\title{
ANÁLISE DE CARACTERÍSTICAS SOCIODEMOGRÁFICAS, CLÍNICAS E DE SOBREVIDA RELACIONADAS A ADULTOS JOVENS PORTADORES DE CÂNCER ORAL
}

\author{
$\underline{\text { Maria Carolina Silva Leite'; }}$ Valéria Souza Freitas²; Marília de Matos Amorim³
}

\author{
1. Bolsista PIBIC/CNPq, Graduando em Odontologia, Universidade Estadual de Feira de Santana, \\ e-mail: carolinaleite26@ hotmail.com \\ 2. Orientador, Departamento de Saúde, Universidade Estadual de Feira de Santana, \\ e-mail: valeria.souza.freitas@gmail.com \\ 3. Mestranda do Programa de Pós-graduação em Saúde Coletiva, Departamento de Saúde, Universidade Estadual \\ de Feira de Santana, e-mail: amorim.mah@hotmail.com
}

PALAVRAS-CHAVE: sobrevida; adultos jovens; câncer oral

\section{INTRODUÇÃO}

A maior incidência do câncer oral ocorre em indivíduos de meia idade a idosos. Porém, estudos retrospectivos, tem demonstardo uma tendência a um aumento do número de casos em adultos jovens (VENTURI et al., 2004; WARNAKULASURIYA, 2009). Alguns atores também sugerem que o câncer da cavidade oral é mais agressivo em adultos jovens quando comparado aos idosos (IAMAROON et al., 2004; WARNAKULASURIYA et al. 2007; CHANG et al., 2013). Desta forma, o objetivo principal deste estudo é descrever as principais características sociodemográficas, clínicas, histológicas, de estadiamento clínico, tratamento e sobrevida em adultos jovens portadores de câncer oral.

\section{MATERIAL E MÉTODOS}

Trata-se de um estudo observacional, longitudinal a partir de um coorte de adultos jovens, com diagnóstico de câncer da cavidade oral, atendidos no período de 2010 a 2016, na UNACON, em Feira de Santana, Bahia. Foram incluídos no estudo todos os indivíduos com o diagnóstico de câncer oral, com idade igual ou inferior a 45 anos. O tempo de sobrevida, em meses, foi definido como o período entre entrada do indivíduo no estudo (data do diagnóstico) até a ocorrência do evento de interesse (óbito pela doença) ou da última consulta. As variáveis foram obtidas dos prontuários médicos. Foi realizada uma análise descritiva de todas as variáveis e para estimar as probabilidades de sobrevida a cada tempo foi utilizado o estimador de Kaplan-Meier. Todas as análises foram realizadas com o programa estatístico SPSS, versão 17.0. O projeto foi aprovado pelo Comitê de Ética em Pesquisa em Seres Humanos (CEP) da UEFS sob Protocolo No 015/2008, CAAE 0015.0.059.000-08.

\section{RESULTADOS}

No período de 2010 a 2016 foram diagnosticados 45 casos de câncer oral em indivíduos com idade igual ou inferior a 45 anos.

A amostra era predominantemente do sexo masculino, com ocupação, raça/cor não branca, analfabetos, moradores de zona urbana e de municípios de grande ou médio porte. Em relação ao estilo de vida, a maioria dos indivíduos era fumantes por tempo igual ou maior a 25 anos e consumiam na sua maior parte fumo de corda. Em relação à ingestão de bebidas alcoólicas, a maior parte da amostra não consumia bebidas alcoólicas. Entre aqueles que bebiam, faziam por tempo igual ou superior a 25 anos, sendo a bebida destilada a mais consumida.

Quanto às variáveis clínicas relacionadas ao câncer oral (Tabela 1), observa-se que boa parte das lesões eram carcinomas escamocelulares(CEC) de língua, com duração entre 6 meses e 1 ano e com gradação histológica bem diferenciada. 
Tabela 1. Distribuição dos indivíduos jovens portadores de câncer oral, segundo variáveis clínicas e histológicas, UNACON, Feira de Santana, BA, 2010 a 2016.

\begin{tabular}{|c|c|c|}
\hline Variáveis clínicas e histológicas & $\begin{array}{l}\text { Frequência absoluta } \\
\text { (n) }\end{array}$ & $\begin{array}{c}\text { Frequência relativa } \\
(\%)\end{array}$ \\
\hline \multicolumn{3}{|l|}{ Localizacão topográfica $(n=44)$} \\
\hline Língua & 16 & 36,4 \\
\hline Lábio superior & 01 & 2,3 \\
\hline Lábio inferior & 02 & 4,5 \\
\hline Assoalho de boca & 04 & 9,1 \\
\hline Palato mole & 02 & 4,5 \\
\hline Amígdala & 03 & 6,8 \\
\hline Mucosa jugal & 03 & 6,8 \\
\hline Palato duro & 04 & 9,1 \\
\hline Gengiva & 02 & 4,5 \\
\hline Glândula submandibular & 04 & 9,1 \\
\hline Glândula parótida & 02 & 4,5 \\
\hline Retromolar & 01 & 2,3 \\
\hline \multicolumn{3}{|l|}{ Tipo histológico da lesão $(n=45)$} \\
\hline Carcinoma escamocelular & 29 & 64,4 \\
\hline Carcinoma adenoide cístico & 03 & 6,7 \\
\hline Carcinoma de células acinares & 01 & 2,2 \\
\hline Adenocarcinoma polimorfo de baixo grau & 03 & 6,7 \\
\hline Carcinoma mioepitelial & 01 & 2,2 \\
\hline Carcinoma mucoepidermoide de baixo grau & 04 & 8,9 \\
\hline Linfoma difuso de células grandes & 01 & 2,2 \\
\hline Rabdomiossarcoma & 01 & 2,2 \\
\hline Carcinossarcoma & 01 & 2,2 \\
\hline Neoplasia maligna fusocelular & 01 & 2,2 \\
\hline \multicolumn{3}{|l|}{ Duração da lesão $(n=28)$} \\
\hline$<6$ meses & 10 & 35,8 \\
\hline 6 meses a 1 ano & 14 & 50,0 \\
\hline 1 a 2 anos & 02 & 7,1 \\
\hline$>2$ anos & 02 & 7,1 \\
\hline \multicolumn{3}{|l|}{ Gradação histológica $(\mathrm{n}=24)$} \\
\hline Pouco indiferenciado & 06 & 25,0 \\
\hline Moderadamente diferenciado & 08 & 33,3 \\
\hline Bem diferenciado & 10 & 41,7 \\
\hline
\end{tabular}

\section{Fonte: Própria}

A Tabela 2 demonstra que quanto ao estadiamento clínico, maior parte dos casos foi diagnosticada no estágio IV $(69,4 \%)$. O tratamento mais utilizado foi a cirurgia $(24,4 \%)$.

Os indivíduos diagnosticados com câncer oral, tiveram uma média do tempo de sobrevida de 92 meses. Em relação ao CEC, dos 29 casos diagnosticados, 26 possuíam informações acerca das datas de diagnóstico e óbito/última consulta, e destes, nove foram a óbito. O tempo médio de sobrevida foi de 36,21 meses (Figura 1). Quanto ao status de sobrevida, de todos os casos estudados $30 \%$ foram a óbito, 25,6\% tiveram remissão parcial da doença (Tabela 2).

Figura 1. Sobrevida em meses dos casos de carcinoma de células escamosas em indivíduos jovens. UNACON, Feira de Santana, BA, 2010 a 2016.

Proporção cumulativa de sobrevida

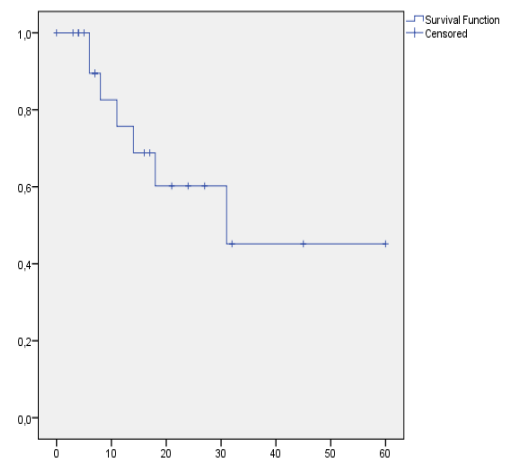

Tempo de sobrevida em meses 
Tabela 2. Distribuição dos indivíduos jovens portadores de câncer oral, segundo estadiamento, tipo de tratamento e status de sobrevida, UNACON, Feira de Santana, BA, 2010 a 2016.

\begin{tabular}{lcc}
\multicolumn{1}{c}{$\begin{array}{c}\text { Variáveis de estadiamento, tratamento } \\
\text { e status de sobrevida }\end{array}$} & $\begin{array}{c}\text { Frequência absoluta } \\
(\mathbf{n})\end{array}$ & $\begin{array}{c}\text { Frequência relativa } \\
(\boldsymbol{\%})\end{array}$ \\
\hline $\begin{array}{l}\text { Estadiamento }(\mathbf{n}=\mathbf{3 5}) \\
\text { Estádio I }\end{array}$ & 03 & 8,3 \\
Estádio II & 01 & 2,8 \\
Estádio III & 07 & 19,4 \\
Estádio IV & 25 & 69,4 \\
Tipo de tratamento (n=41) & 10 & 24,4 \\
Cirurgia & 02 & 4,9 \\
Radioterapia & 03 & 7,3 \\
Quimioterapia & 09 & 22,0 \\
Cirurgia, quimioterapia e radioterapia & 05 & 12,2 \\
Cirurgia e quimioterapia & 09 & 22,0 \\
Quimioterapia e radioterapia & 03 & 7,3 \\
Não realizou tratamento & & 10,3 \\
Status de sobrevida (n=39) & 04 & 25,6 \\
Sem evidência & 03 & 15,4 \\
Remissão parcial & 10 & 15,4 \\
Doença estável & 06 & 2,6 \\
Doença em progressão & 06 & 30,8 \\
Fora de possibilidade terapêutica & 01 & \\
Óbito & 12 & \\
\hline Fonte: Própria & & \\
\hline
\end{tabular}

Fonte: Própria

\section{DISCUSSÃO}

A maioria dos casos de câncer oral ocorreu em indivíduos do sexo masculino, não brancos e com baixa escolaridade, estando tais achados de acordo com outros estudos (LOSIGUEMBAROVSKI et al., 2009; SANTOS et al., 2009).

No presente estudo, a maior parte dos indivíduos diagnosticados com CEC apresentava baixa escolaridade. Estes achados são consistentes com outros estudos (PEREIRA et al., 2003, MELO et al., 2010). A maioria dos indivíduos do nosso estudo eram fumantes crônicos, porém, a maior parte da amostra não relatou o consumo de bebidas alcóolicas. Esses achados estão parcialmente de acordo com outros autores (MACKENZIE et al., 2000). Estes fatores de risco relacionados à doença em indivíduos acima de 40 anos, talvez não sejam tão relevantes no caso da doença em adultos jovens (VENTURINI et al., 2004), uma vez que o tempo de exposição a esses fatores é muito curto para produzir transformações malignas (HIROTA et al., 2006). Para alguns autores, a infecção pelo Papiloma Vírus Humano (HPV) em adultos jovens pode ser uma possível explicação para a etiologia da doença (KAMINAGAKURA et al., 2010).

A maioria das neoplasias malignas diagnosticadas no nosso estudo correspondiam ao CEC. Para Barnes e colaboradores (2005), mais de 90\% das neoplasias malignas que ocorrem na cavidade oral e orofaringe são deste tipo histológico. Quanto a localização, a maior parte foi diagnosticada em língua, corroborando com outros autores (SOUNDRY et al., 2010).

No presente estudo, a maioria dos tumores foi diagnosticado em estágio avançado da doença (estágios III e IV). No estudo de Soundry et al. (2010), que comparou pacientes com CEC de língua em pacientes jovens e idosos, observou-se que nos jovens os estágios III e IV foram mais freqüentes, diferente dos idosos (estadiamento I e II).

A maioria dos indivíduos do nosso estudo foram tratados exclusivamente por cirurgia $(24,4 \%)$. A ressecção cirúrgica tem sido descrita como o tratamento de escolha, semelhante ao tratamento em idosos (HO et al., 2008; HILLY et al., 2013; MORAIS et al., 2017).

O tempo médio de sobrevida dos indivíduos com câncer oral do nosso estudo foi de 92 meses. A maioria dos indivíduos com CEC foi a óbito (30\%), tendo um tempo médio de 
sobrevida de 36,21 meses. Estudos com adultos jovens têm mostrado que o diagnóstico realizado tardiamente dificulta o prognóstico e o tempo de sobrevida dos pacientes (SOUDRY et al., 2010; HILLY et al., 2013).

Os nossos resultados devem ser observados levando em consideração suas limitações, já que foi um estudo exploratório preliminar, que contou com uma amostra pequena, impossibilitando desta forma estimar os efeitos de cada variável na sobrevida dos indivíduos.

\section{CONCLUSÃO}

Neste estudo foram encontrados 45 casos de câncer oral em jovens com idade igual ou inferior a 45 anos. A maioria dos indivíduos eram homens, não brancos, com baixa escolaridade, moradores da zona urbana e tabagistas crônicos. Os tumores eram predominantemente CEC em língua, diagnosticados em estágios avançados e tratados principalmente por cirurgia. A maioria dos casos de CEC foram a óbito, tendo como tempo médio de sobrevida de 36,21 meses.

\section{REFERÊNCIAS}

1. CHANG, T. S. et al. Impact of young age on the prognosis for oral cancer: A population-based study in Taiwan. PLoS ONE, v. 8, n.9, p.e75855, 2013.

2. KAMINAGAKURA, E. et al. Case-control study on prognostic factors in oral squamous cell carcinoma in young patients. Head Neck, v. 32, n. 11, p. 1460-6, 2010.

3. HILLY, O. et al. Carcinoma of the oral tongue in patients younger than 30 years: Comparison with patients older than 60 years. Oral Oncology, v. 49, p. 987, 2013.

4. HIROTA, S. K.; MIGLIARI, D. A.; SUGAYA, N. N. Carcinoma epidermóide oral em paciente jovem-Relato de caso e revisão da literatura. Anais Brasileiros de Dermatologia, v. 81, n. 3, p. 251-4, 2006.

5. HO, H. C. et al: Squamous cell carcinoma of the oral cavity in young patients: A matched-pair analysis. Eur Arch Otorhinolaryngol, v. 265, p. S57, 2008.

6. IAMAROON, A. et al. Analysis of 587 cases of oral squamous cell carcinoma in northern Thailand with a focus on young people. International Journal of Oral and Maxillofacial Surgery, v. 33, n. 1, p. 84-88, 2004.

7. LOSI-GUEMBAROVSKI, R. et al. Oral carcinoma epidemiology in Paraná State, Southern Brazil. Cadernos de Saúde Pública, v. 25, p. 393-2000, 2009.

8. MACKENZIE, J. et al. Increasing incidence of oral cancer amongst young persons: what is the aetiology? Oral Oncology, v. 36, n. 4, p. 397-399, 2000.

9. MELO, L. C. Perfil epidemiológico de casos incidentes de câncer de boca e faringe. Revista Gaúcha Odontologia, v. 58, n. 3, p. 351-355, 2010.

10. MORAIS, E F et al. Prognostic factors of oral squamous cell carcinoma in young patients: a systematic review. Journal of Oral and Maxillofacial Surgery, 2017, in press.

11. PEREIRA, L. A. et al. Epidemiologia do câncer bucal em Barretos, Revista Brasileira Cirurgia Cabeça Pescoço, v. 31, n. 2, p. 35-39, 2003.

12. SANTOS, L. C. O. et al. Câncer bucal: amostra populacional do Estado de Alagoas em hospital de referência. Brazilian Journal of Otorhinolaryngology, v. 75, p. 524-529, 2009.

13. SOUDRY, E. et al. Squamous cell carcinoma of the oral tongue in patients younger than 30 years: Clinicopathologic features and outcome. Clin Otolaryngol, v. 35, p. 307, 2010.

14. WARNAKULASURIYA, S. Global epidemiology of oral and oropharyngeal cancer. Oral Oncology, v. 45, n. 4, p. 309-316, 2009.

15. WARNAKULASURIYA, S.; JOHNSON, N.W.; VAN DER WAAL, I. Nomenclature and classification of potentially malignant disorders of the oral mucosa. Journal of Oral Pathology \&Medicine,v. 36, n. 10, p. 575-580, 2007.

16. VENTURI, B. M. et al. Carcinoma de células escamosas da cavidade oral em pacientes jovens e sua crescente incidência: revisão de literatura. Revista Brasileira Otorrinolaringologia, v.70, n.5, p.679-86, 2004. 